\title{
NOTE ON VOLTERRA AND FREDHOLM PRODUCTS OF SYMMETRIC KERNELS*
}

BY L. M. BLUMENTHAL

1. Introduction. $\dagger$ The purpose of this paper is to establish two theorems concerning the Volterra and the Fredholm products of two continuous, symmetric functions. These two theorems were obtained in the course of an investigation that had as its object the determination of the existence of permutable functions (first kind) of the second order; that is, the determination of functions $K_{1}(x, y), K_{2}(x, y)$ such that

$$
K_{1} K_{2} K_{2} K_{1}=K_{2} K_{1} K_{1} K_{2}
$$

but

where

$$
K_{1} K_{2} \neq K_{2} K_{1}
$$

$$
K_{1} K_{2}=\int_{x}^{y} K_{1}(x, t) K_{2}(t, y) d t .
$$

To satisfy (1), $\ddagger$ it is sufficient to find two functions $K_{1}, K_{2}$ such that

$$
K_{1} K_{2}=-K_{2} K_{1},
$$

for, composing (on the right) the left-hand member with $K_{2} K_{1}$, and the right-hand member with its equal, $-K_{1} K_{2}$, we obtain (1). If two functions satisfy (2), we shall call them skew permutable. Assume, now, that $K_{1}(x, y), K_{2}(x, y)$ are symmetric functions of their arguments. We have the following lemma.

Lemma. The Volterra product of two symmetric functions is itself a symmetric function if and only if the two functions are skew permutable.

Let

$$
K(x, y)=\int_{x}^{y} K_{1}(x, \xi) K_{2}(\xi, y) d \xi=K_{1} K_{2} ;
$$

* Presented to the Society, September 9, 1931.

$\dagger$ I wish to thank G. C. Evans for suggestions given me during the preparation of this paper.

$\ddagger$ In this part of the paper we are concerned entirely with Volterra composition. 
then

$$
K(y, x)=\int_{y}^{x} K_{1}(y, \xi) K_{2}(\xi, x) d \xi,
$$

and since $K_{1}$ and $K_{2}$ are symmetric functions,

$$
K(y, x)=-\int_{x}^{y} K_{2}(x, \xi) K_{1}(\xi, y) d \xi=-K_{2} K_{1}
$$

Hence $K(x, y)=K(y, x)$, if and only if $K_{1} K_{2}=-K_{2} K_{1}$, that is, if and only if the functions $K_{1}, K_{2}$ are skew permutable.

Hence the problem of finding two symmetric functions whose Volterra product is symmetric is equivalent, by means of the Lemma, to finding two skew permutable functions.

Before treating the general case, we show that if a continuous function is skew permutable with unity, then the function is identically zero.*

If $K_{2}(x, y)$ is such a function, then

$$
\int_{x}^{y} K_{2}(\xi, y) d \xi=-\int_{x}^{y} K_{2}(x, \xi) d \xi \text {. }
$$

Calling the common value of the two integrals $\phi(x, y)$, we have

$$
\frac{\partial \phi}{\partial x}=-K_{2}(x, y), \quad \frac{\partial \phi}{\partial y}=-K_{2}(x, y),
$$

and $\phi(x, y)$ must satisfy the equation

$$
\frac{\partial \phi}{\partial x}-\frac{\partial \phi}{\partial y}=0
$$

that is,

$$
\phi=\phi(y+x) \text {. }
$$

But $\phi(x, x) \equiv 0$, hence $\phi(y+x) \equiv 0, \dagger$ and therefore $K_{2}(x, y)$ is identically zero. We now state the following theorem.

* This is in marked contrast to functions permutable with unity. These are functions of $(y-x)$, the so-called functions of closed cycle of Volterra.

$\dagger$ Consider $\phi=\phi(y+x)$. Along the lines $x+y=c$ in the $X Y$ plane, $\phi \equiv c$. But along the line $y=x$ in this plane, $\phi \equiv 0$. Hence $\phi(y+x)$ vanishes identically. 
THEOREM 1. If the Volterra product of two continuous, symmetric functions, $K_{1}(x, y), K_{2}(x, y)$, where $K_{1}$ is of the first order, with continuous first partial derivatives and with $\partial^{2} K_{1} / \partial x \partial y$ continuous, is a symmetric function, then one of the functions is identically zero.

Now if a function $F(x, y)$ is of the first order, we may form the function

$$
F_{1}\left(x_{1}, y_{1}\right)=a\left(x_{1}\right) b\left(y_{1}\right) F\left[m\left(x_{1}\right), m\left(y_{1}\right)\right],
$$

where we have written

$$
x=m\left(x_{1}\right), \quad y=m\left(y_{1}\right), m^{\prime}\left(x_{1}\right) \neq 0,
$$

and the functions $a$ and $b$ satisfy the relation

$$
a\left(x_{1}\right) b\left(x_{1}\right)=m^{\prime}\left(x_{1}\right) .
$$

It may be shown* that if

$$
a=e^{-\mu}, \quad b=\frac{1}{F(x, x)} e^{\mu}
$$

where

$$
\mu=\int \frac{\left(\frac{\partial F}{\partial x}\right)_{y=x}}{F(x, x)} d x
$$

then $F_{1}\left(x_{1}, y_{1}\right)$ is such that

$$
F_{1}\left(x_{1}, x_{1}\right)=1,\left(\frac{\partial F_{1}}{\partial x_{1}}\right)_{y_{1}=x_{1}}=\left(\frac{\partial F_{1}}{\partial y_{1}}\right)_{y_{1}=x_{1}}=0 .
$$

We say that the function $F_{1}$ is in canonical form.

We shall assume that our function $K_{1}(x, y)$ is in canonical form. We prove our theorem by showing that if $K_{2}(x, y)$ is a continuous function, skew permutable with $K_{1}$, then $K_{2}$ vanishes identically. $\uparrow$ Then an application of the Lemma yields the theorem.

* See, for example, Volterra et Pérès, Leçons sur la Composition, Paris, 1924, p. 38.

$\dagger$ To show this it is unnecessary to assume $K_{1}, K_{2}$ symmetric. Their symmetry enters, however, in applying the Lemma. 
Suppose that $K_{2}(x, y)$ is a continuous function satisfying the condition $K_{1} K_{2}=-K_{2} K_{1}$. Calling the common value of each side $\phi$, we have

$$
\phi(x, y)=\int_{x}^{y} K_{1}(x, \xi) K_{2}(\xi, y) d \xi=-\int_{x}^{y} K_{2}(x, \xi) K_{1}(\xi, y) d \xi,
$$

with $\phi(x, x) \equiv 0$.

Differentiating, and recalling that $K_{1}(x, x)=1$, we obtain

$$
\begin{aligned}
& \frac{\partial \phi}{\partial x}=-K_{2}(x, y)+\int_{x}^{y} \frac{\partial K_{1}(x, \xi)}{\partial x} K_{2}(\xi, y) d \xi, \\
& \frac{\partial \phi}{\partial y}=-K_{2}(x, y)-\int_{x}^{y} K_{2}(x, \xi) \frac{\partial K_{1}}{\partial y}(\xi, y) d \xi .
\end{aligned}
$$

Solving these two Volterra integral equations in $K_{2}(x, y)$ we get

$$
\begin{aligned}
& K_{2}(x, y)=-\frac{\partial \phi}{\partial x}-\int_{x}^{y} k(x, \xi) \frac{\partial \phi(\xi, y)}{\partial \xi} d \xi, \\
& K_{2}(x, y)=-\frac{\partial \phi}{\partial y}+\int_{x}^{y} \frac{\partial \phi(x, \xi)}{\partial \xi} g(\xi, y) d \xi,
\end{aligned}
$$

where $k(x, y), g(x, y)$ are the resolvent kernels for $\partial K_{1} / \partial x$ and $\partial K_{1} / \partial y$ respectively.

Performing an integration by parts in each of the last two equations, and taking into account that $\left(\partial K_{1} / \partial x\right)_{y=x}$ $=\left(\partial K_{1} / \partial y\right)_{y=x}=0$, we have

$$
\begin{aligned}
& K_{2}(x, y)=-\frac{\partial \phi}{\partial x}+\int_{x}^{y} \frac{\partial k(x, \xi)}{\partial \xi} \phi(\xi, y) d \xi, \\
& K_{2}(x, y)=-\frac{\partial \phi}{\partial y}-\int_{x}^{y} \phi(x, \xi) \frac{\partial g(\xi, y)}{\partial \xi} d \xi .
\end{aligned}
$$

From these two equations, and the relation*

$$
\frac{\partial k(x, y)}{\partial y}=\frac{\partial g(x, y)}{\partial x}=\eta(x, y)
$$
p. 40.

* This is due to Pérès; see Volterra et Pérès, Leçons sur la Composition, 
we finally obtain $\phi$, given by means of the integro-differential equation

$$
\frac{\partial \phi}{\partial x}-\frac{\partial \phi}{\partial y}=\int_{x}^{y}[\phi(x, \xi) \eta(\xi, y)+\eta(x, \xi) \phi(\xi, y)] d \xi .
$$

If we denote the right-hand member by $\varepsilon(x, y)$, we have

$$
\frac{\partial \phi}{\partial x}-\frac{\partial \phi}{\partial y}=\varepsilon(x, y),
$$

from which

$$
\phi(x, y)=\int_{0}^{x} \varepsilon(x, y) d x+\theta(y+x)
$$

with $\theta$ an arbitrary function. But $\phi(x, x) \equiv 0$, whence, since $\mathcal{E}(x, x) \equiv 0$, we have $\theta(2 x) \equiv 0$. Then $\theta(y+x) \equiv 0$ and (3) becomes

$$
\phi(x, y)=\int_{0}^{x} \mathcal{E}(x, y) d x,
$$

a homogeneous Volterra integral equation for $\phi$. But this equation has as its only continuous solution $\phi \equiv 0$. Then $K_{2} \equiv 0$, and the theorem is proved.

COROLLARY. The theorem is valid for functions $K_{1}(x, y)$ of any definite order.

2. Fredholm Composition. In this section we deal exclusively with composition of the second kind.* It is well known that if $K(x, y)$ is a symmetric kernel, then all of its iterated kernels, $K_{n}(x, y)$, are symmetric, and since

$$
K_{n}=\int_{a}^{b} K(x, \xi) K_{n-1}(\xi, y) d \xi,
$$

we have an example of two symmetric functions whose Fredholm product is likewise symmetric. We seek now to characterize those symmetric functions whose Fredholm product is also symmetric, at least in the case of both functions having only a finite number of characteristic values.

* Volterra, Questioni generali sulle equazioni integrali ed integro-differenziali, Rendiconti dei Lincei, ${ }^{\text {er }}$ sem., 1910, p. 178. See also, Volterra, Leçons sur les Fonctions des Lignes, Paris, 1913, p. 179. 
Let $K(x, y), G(x, y)$ be two continuous, symmetric kernels; then

$$
\begin{aligned}
K G(x, y) & =\int_{a}^{b} K(x, \xi) G(\xi, y) d \xi \\
K G(y, x) & =\int_{a}^{b} K(y, \xi) G(\xi, x) d \xi \\
& =\int_{a}^{b} G(x, \xi) K(\xi, y) d \xi, \\
& =G K(x, y) .
\end{aligned}
$$

Thus the Fredholm product of two symmetric kernels is symmetric if and only if $K G=G K$; that is, if and only if the kernels $K, G$ are permutable of the second kind.

Now Volterra has shown* that the problem of permutability of the second kind can be reduced (at least in the case of kernels having a finite number of characteristic constants) to the problem of permutability of square matrices. In place of applying the complicated general theory (in which, of course, no hypothesis of symmetry on the part of the kernels is made) we shall obtain the characterization sought by methods more germane to the problem.

If, now, $K(x, y), G(x, y)$ are symmetric kernels such that

$$
P(x, y)=\int_{a}^{b} G(x, \xi) K(\xi, y) d \xi=P(y, x),
$$

then it is evident that $\int_{a}^{b} G_{r}(x, \xi) K_{s}(\xi, y) d \xi$ is also a symmetric function. Suppose $K(x, y), G(x, y)$ admit the distinct characteristic constants $\lambda_{1}, \lambda_{2}, \cdots, \lambda_{k}$ and $\mu_{1}, \mu_{2}, \cdots, \mu_{g}$ respectively. Then we may write

$$
\begin{aligned}
& K_{r}(x, y)=\sum_{i=1}^{k} \frac{\phi_{i}(x) \phi_{i}(y)}{\lambda_{i}{ }^{r}}, \quad(r=1,2,3, \cdots), \\
& G_{s}(x, y)=\sum_{j=1}^{g} \frac{\theta_{j}(x) \theta_{j}(y)}{\mu_{j}{ }^{s}}, \quad(s=1,2,3, \cdots),
\end{aligned}
$$

* Volterra, Lȩons sur les Fonctions des Lignes, Chapter 12. 
where $\phi_{i}, \theta_{j}$ are the characteristic functions of $K$ and $G$ respectively, corresponding to the characteristic values $\lambda_{i}$ and $\mu_{j}$. Now, in order that the Fredholm product $K G$ be symmetric, it is necessary that

$$
\int_{a}^{b} K_{r}(x, \xi) G_{s}(\xi, y) d \xi=\int_{a}^{b} K_{r}(y, \xi) G_{s}(\xi, x) d \xi
$$

for all positive integers $r, s$. Writing

$$
\begin{aligned}
\Phi_{i}(x, y) & =\phi_{i}(x) \phi_{i}(y), \\
\Theta_{j}(x, y) & =\theta_{j}(x) \theta_{j}(y),
\end{aligned}
$$

we have

(6) $\int_{a}^{b} K_{r}(x, \xi) G_{s}(\xi, y) d \xi=\sum_{i=1}^{k} \sum_{j=1}^{g} \frac{\int_{a}^{b} \Phi_{i}(x, \xi) \Theta_{j}(\xi, y) d \xi}{\lambda_{i}{ }^{r} \mu_{j}{ }^{s}}$;

and hence, substituting in (4),

$$
\sum_{i=1}^{k} \sum_{j=1}^{g}\left[\frac{\int_{a}^{b} \Phi_{i}(x, \xi) \Theta_{j}(\xi, y) d \xi-\int_{a}^{b} \Phi_{i}(y, \xi) \Theta_{j}(\xi, x) d \xi}{\lambda_{i}{ }^{r} \mu_{j}{ }^{s}}\right]=0
$$

for all positive integral values of $r, s$.

We write the above equations in the form

$$
\begin{aligned}
\sum_{i=1}^{k} \frac{1}{\lambda_{i}^{r}}\left[\sum_{j=1}^{g} \frac{\int_{a}^{b} \Phi_{i}(x, \xi) \Theta_{j}(\xi, y) d \xi-\int_{a}^{b} \Phi_{i}(y, \xi) \Theta_{j}(\xi, x) d \xi}{\mu_{j}^{s}}\right] \\
=\sum_{i=1}^{k} \frac{1}{\lambda_{i}^{r}} X_{i}=0,
\end{aligned}
$$

and agree to hold $s$ fixed, while $r$ takes on the values $1,2, \cdots$. We obtain in this way an infinite number of homogeneous equations that the $k$ functions $X_{i}$ must satisfy. Consider the first $k$ of these equations. The determinant of the coefficients is the Vandermond determinant $\left|1 / \lambda_{i}{ }^{r}\right|,(i, r=1,2, \cdots, k)$, of order $k$. This determinant is not zero, and hence the first $k$ equations of the set have no solution other than $X_{i}=0,(i=1,2, \cdots, k)$. 
Whence this is the only solution of the complete set of equations. Thus

$$
\begin{aligned}
\sum_{j=1}^{g} \frac{1}{\mu_{j}^{s}}\left[\int_{a}^{b} \Phi_{i}(x, \xi) \Theta_{j}(\xi, y) d \xi\right. & \\
& \left.-\int_{a}^{b} \Phi_{i}(y, \xi) \Theta_{j}(\xi, x) d \xi\right]=\sum_{j=1}^{g} \frac{1}{\mu_{j}^{s}} \Gamma_{j}=0 .
\end{aligned}
$$

Again we have an infinite set of equations in $g$ functions $\Gamma_{j}$ and the determinant of the coefficients appearing in the first $g$ of the equations is the non-vanishing Vandermond determinant $\left|1 / \mu_{j}^{s}\right|,(j, s=1,2, \cdots, g)$. Hence the only solution of the system is $\Gamma_{j}=0$, and we have

$$
\int_{a}^{b} \Phi_{i}(x, \xi) \Theta_{j}(\xi, y) d \xi=\int_{a}^{b} \Phi_{i}(y, \xi) \Theta_{j}(\xi, x) d \xi,
$$

for each $i=1,2, \cdots, k$ and $j=1,2, \cdots, g$, as the necessary conditions that the Fredholm product $K G$ be symmetric. These conditions are evidently sufficient, as an inspection of (6) assures us that if these conditions are satisfied, then

$$
\int_{a}^{b} K_{r}(x, \xi) G_{s}(\xi, y) d \xi=\int_{a}^{b} K_{r}(y, \xi) G_{s}(\xi, x) d \xi .
$$

We state the foregoing in the form of the following theorem.

THEOREM 2. In order that the Fredholm product of two symmetric kernels, admitting only a finite number of characteristic constants, be symmetric, it is necessary and sufficient that the conditions

$$
\int_{a}^{b} \Phi_{i}(x, \xi) \Theta_{j}(\xi, y) d \xi=\int_{a}^{b} \Phi_{i}(y, \xi) \Theta_{j}(\xi, x) d \xi
$$

be satisfied for each $i=1,2, \cdots, k ; j=1,2, \cdots, g$.

It is evident that these conditions are highly restrictive. Substituting from (5), conditions (8) reduce to

$$
c_{i j} \phi_{i}(x) \theta_{j}(y)=c_{i j} \theta_{j}(x) \phi_{i}(y),
$$

where

$$
c_{i j}=\int_{a}^{b} \phi_{i}(\xi) \theta_{j}(\xi) d \xi
$$


Thus the conditions are satisfied if and only if the characteristic functions of $K$ and $G$ are orthogonal, or satisfy the relations

so that

$$
\frac{\phi_{i}(x)}{\theta_{j}(x)}=\frac{\phi_{i}(y)}{\theta_{j}(y)}=\text { a constant, say } k_{i j}
$$

$$
\phi_{i}(x)=k_{i j} \theta_{j}(x) .
$$

That the class of functions $G(x, y)$, symmetric, and forming with a symmetric function $K(x, y)$ a symmetric Fredholm product, is so intimately related to the function $K(x, y)$ in the case of each of the functions having a finite number of characteristic constants, is a result that (it appears to the writer) could hardly have been anticipated. This is even more striking when the result is viewed as a theorem giving necessary and sufficient conditions for the permutability of the second kind of two functions satisfying the conditions of the theorem. In addition to exhibiting a fact that does not seem to have found a place in the literature of permutable functions of the second kind, the simplicity of the method used is in marked contrast to the more complicated procedure usual in treatments of this subject.

It might appear at first that using the well known development of the iterated kernels in terms of the characteristic functions

$$
\begin{array}{rlrl}
K_{r}(x, y) & =\sum_{i=1}^{\infty} \frac{\phi_{i}(x) \phi_{i}(y)}{\lambda_{i}{ }^{r}}, & & (r \geqq 2), \\
G_{s}(x, y)=\sum_{j=1}^{\infty} \frac{\theta_{j}(x) \theta_{j}(y)}{\mu_{j}{ }^{s}}, & & (s \geqq 2),
\end{array}
$$

both series converging absolutely and uniformly, we might treat the case for which $G$ and $K$ admit infinitely many characteristic values by means of an obvious extension of the previous analysis. That this is not the case is due to the fact that when we consider the infinite set of homogeneous equations in an infinity of unknowns that is the analog of the set of equations (7), the infinite Vandermond determinant

$$
\left|\frac{1}{\lambda_{i}^{r}}\right|, \quad\left(\begin{array}{l}
r=2,3, \cdots \\
i=1,2, \cdots
\end{array}\right),
$$


converges to zero. This is immediate upon applying two theorems due to T. Gazzaniga." Indeed, these theorems are sufficient to establish in general that the infinite Vandermond determinant formed by taking the reciprocals of the infinitely many zeros of a transcendental integral function converges to zero. Thus, the foregoing method is not well adapted to kernels with an infinite number of characteristic values.

Since we have shown that the problem is equivalent to functions $K, G$ being permutable of the second kind, the general theory may be applied to determine the conditions under which this takes place. This is a problem of much difficulty. It is possible that modifying Volterra's definition of composition so that

$$
E(x, y)=K(x, y)+G(x, y)+\int_{x}^{y} K(x, \xi) G(\xi, y) d \xi,
$$

is defined to be the composition of $K$ and $G$ (which leaves the condition of permutability unaltered) may make the problem capable of being handled by means of the algebra of functions introduced and developed by Griffith C. Evans. $\dagger$

The Rice Institute

* Sui determinanti d'ordine infinito, Annali di Matematica, (2), vol. 26, p. 205; Intorno ad un tipo di determinanti d'ordine infinito, ibid., (3), vol. 1, pp. 83-94.

† See his Cambridge Colloquium Lectures, Functionals and their Applications, 1918, p. 119; see also Sopra l'algebra delle funzioni permutabili, Atti dei Lincei, vol. 8 (1911), p. 6. 